\title{
Seeds as perfect factories for developing sustainable agriculture
}

\author{
Gwyneth Ingram ${ }^{1} \cdot$ Helen North ${ }^{2} \cdot$ Loïc Lepiniec $^{2}$ (I)
}

Received: 16 May 2018 / Accepted: 5 June 2018 / Published online: 22 June 2018

c) Springer-Verlag GmbH Germany, part of Springer Nature 2018

Angiosperm seeds are perfect biological factories for producing food, feed and industrially valuable molecules. They have evolved to accumulate a huge variety of nutritional and protective molecules in a stable (dry and largely quiescent) environment that can be stored, in some cases for many years, allowing embryo growth to be resumed when placed under favourable conditions. These characteristics have made seeds the cornerstone of human agriculture, and today they directly provide the majority of the calories consumed by an average human being (http://www.fao.org/seeds /en/, www.fao.org/3/a-i4691e.pdf). They are, in addition, a key component of many feedstuffs used in the production of animal-derived products. The potential of seeds as bioreactors for the production of high-value heterologous proteins (for example antibodies) has already been identified (Lau and Sun 2009; Tschofen et al. 2016; Weichert et al. 2016; Sabalza et al. 2013; Häkkinen et al. 2016). As a result understanding and optimizing the accumulation and storage of key molecules in seeds, and identifying novel and innovative uses for seed-derived products, represent important strategies in the drive both for increased agricultural efficiency and sustainability, and in the quest for novel biotechnological solutions for the production of high-value molecules. This collection of reviews aims to highlight important new findings in seed biology and pinpoint potentially novel strategies towards this end.

Communicated by L. Lepiniec, H. North, G. Ingram.

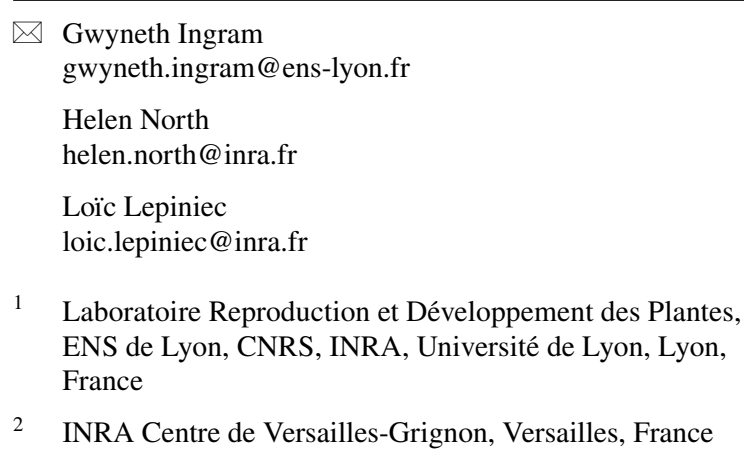

2 INRA Centre de Versailles-Grignon, Versailles, France

Seeds are not simple organs. They are compound structures composed of several genetically distinct compartments, nested one inside another (i.e. embryo, endosperm and maternal tissues), which develop in a highly coordinated fashion to ensure the survival of the embryonic plant. As a result mastering the "seed factory" requires of researchers not only an understanding of the biochemical pathways involved in the synthesis of particular molecules but, in addition, a knowledge of the compartmentalization of these pathways within seed tissues, the fluxes of key metabolites between these compartments, and the roles that different compartments play in seed biology over and above their storage functions. Importantly, seed structures are not only complex, but also remarkably diverse, particularly in terms of which tissues are used to stock the energy-rich storage compounds (lipids, complex carbohydrates and proteins) that make seeds such a nutritionally important resource. Understanding and manipulating seed metabolism therefore requires a species-adapted appreciation of inter-compartmental fluxes and compartment-specific metabolism. Finally, a detailed and holistic comprehension of basic seed development is indispensable in order to allow the control of key seed traits, such as the relative size of different storage compartments, or absolute seed size (both of which directly affect seed composition), to be dissected.

The main building blocks for all seed metabolic processes are amino acids and sugars imported into the seed from maternal source tissues via the phloem and xylem. In this issue Karmann and colleagues discuss and contrast, in detail, the process of unloading of these molecules from the vasculature in Arabidopsis. Their review, together with those of Aguirre et al. and Lu and Magnani, also addresses multiple aspects of the subsequent distribution of these critical molecules through both symplastic and apoplastic pathways, to the various tissues of the seed. Tissuespecific inter-conversions of transportable building blocks (for example via the metabolism of aspartate family amino acids, reviewed by Wang et al., or the inter-conversion of hexoses and disaccharides, reviewed by Aguirre et al.) may 
significantly complicate our understanding of the fluxes occurring between specific compartments.

Inter-compartmental fluxes are not only controlled by transport capacity, but also the presence of physicochemical gradients, which are, in part, maintained by the sequestration and conversion of transportable molecules. As a result the metabolic pathways active in specific compartments play important roles in determining not only seed composition, but also metabolite fluxes. In seeds, metabolites are unloaded into the maternal tissues enveloping the zygotic compartments (Karmann et al.). They either transit through these maternal tissues to the zygotic endosperm and embryo, or can be metabolized into nutritionally and industrially important molecules en route. Seed coat metabolism and the industrial applications of the diverse polymers that accumulate in this somewhat neglected compartment are reviewed in depth by Francoz et al. in this special issue and are also addressed by Aguirre and colleagues. The evolutionary biology, development and metabolism of another often overlooked maternal tissue, the nucellus, which is largely eliminated during the development of many seeds, but which can develop into a storage tissue somewhat analogous to the zygotic endosperm in the so-called perispermic seeds such as Quinoa, are also addressed in an original review by $\mathrm{Lu}$ and Magnani.

Several of the reviews in this issue (Zhang et al., Aguirre et al., Baud) address the biosynthesis of key complex carbohydrates in both the endosperm and embryo, emphasizing the important differences in metabolite partitioning that occur between seeds with large storage-adapted endosperms (such as cereals), compared with exalbuminous species, in which the endosperm performs mainly transient functions, while the embryo plays a key role in energy storage (such as leguminous seeds and oilseeds). This partitioning is complicated further by the accumulation of seed coat metabolites, which also impact carbon availability for the production of complex carbohydrates (Francoz et al.). In addition to starch metabolism, and as highlighted for maternal tissues, these authors underline the increasing attention being directed to cell-wall polysaccharides as important sources not only of dietary fibre, but also of other nutritionally and industrially important compounds. In an extremely comprehensive and very complementary review, Baud elucidates new findings not only regarding the cellular and biochemical mechanisms underlying the biosynthesis of the staggering range of lipids synthesized in various seeds, but also how lipid metabolism and carbohydrate metabolism interact in the developing seed during storage compound accumulation. He brings into sharp focus the multiple potential industrial applications of many of these highly diverse molecules.
Harnessing the metabolic capacities of seed compartments requires an understanding of the genetic regulation of the growth and subsequent fate of each compartment, as well as the control of the expression of the components of the enzymatic pathways implicated in key biosynthetic pathways. Strategies for increasing seed size, which can increase both grain yield and quality, are addressed in the special issue by $\mathrm{Li}$ et al. who have reviewed the genetic control of this trait in rice. Zhang et al., in detailing genetic and physiological defects identified in the Opaque mutants of maize, have highlighted not only the importance of key pathways for the biosynthesis and stabilization of storage compounds in the maize endosperm, but also the transcriptional control of these processes by key endosperm-specific transcriptional regulators. Finally, advances in understanding the roles and regulation of the LAFL transcription factors, which control seed-specific processes, and in particular the capacity of seed compartments to undergo the maturation process that enables the stable storage of nutrient stores, are addressed in a thought-provoking review by Lepiniec et al.

This special issue brings together the views of experts in the field who draw attention to recent findings in fundamental seed biology, novel breakthroughs in the manipulation of seed composition and structure and potential industrial applications for seed-derived polymers. Interestingly, authors have also pinpointed important technical sticking points, such as a lack of compartment-specific promoters, and a deficit in the understanding of the global effects of the targeted perturbation of specific metabolic pathways, which could orient the direction of future research in this exciting field.

\section{References}

Häkkinen ST, Nuutila AM, Ritala A (2016) Modifying seeds to produce proteins. In: Yada RY (ed) Proteins in food processing, 2nd edn. Woodhead Publishing, Sawston, pp 413-441

Lau OS, Sun SS (2009) Plant seeds as bioreactors for recombinant protein production. Biotechnol Adv 27:1015-1022

Sabalza M, Vamvaka E, Christou P, Capell T (2013) Seeds as a production system for molecular pharming applications: status and prospects. Curr Pharm Des 19:5543-5552

Tschofen M, Knopp D, Hood E, Stoger E (2016) Plant molecular farming: much more than medicines. Ann Rev Anal Chem (Palo Alto Calif) 9:271-294

Weichert N, Hauptmann V, Helmold C, Conrad U (2016) Seed-specific expression of spider silk protein multimers causes long-term stability. Front Plant Sci 7:6 